\title{
Improvement of outcome prediction of hospitalized patients with COVID-19 by a dual marker strategy using high-sensitive cardiac troponin I and copeptin
}

\author{
Christoph C. Kaufmann ${ }^{1}$ (D) Amro Ahmed ${ }^{1}$. Mona Kassem ${ }^{1} \cdot$ Matthias K. Freynhofer $^{1} \cdot$ Bernhard Jäger $^{1}$. \\ Gabriele Aicher $^{2}$ · Susanne Equiluz-Bruck ${ }^{3}$ - Alexander O. Spiel ${ }^{4}$. Florian Vafai-Tabrizi ${ }^{5}$ - Michael Gschwantler ${ }^{6,7}$. \\ Peter Fasching ${ }^{8} \cdot$ Johann Wojta ${ }^{9,10,11} \cdot$ Evangelos Giannitsis $^{12} \cdot$ Kurt Huber $^{1,7,11}$
}

Received: 25 May 2021 / Accepted: 2 November 2021 / Published online: 15 November 2021

(c) The Author(s), under exclusive licence to Springer-Verlag GmbH Germany 2021

\begin{abstract}
Background COVID-19 has been associated with a high prevalence of myocardial injury and increased cardiovascular morbidity. Copeptin, a marker of vasopressin release, has been previously established as a risk marker in both infectious and cardiovascular disease.

Methods This prospective, observational study of patients with laboratory-confirmed COVID-19 infection was conducted from June 6th to November 26th, 2020 in a tertiary care hospital. Copeptin and high-sensitive cardiac troponin I (hs-cTnI) levels on admission were collected and tested for their association with the primary composite endpoint of ICU admission or 28-day mortality.

Results A total of 213 eligible patients with COVID-19 were included of whom 55 (25.8\%) reached the primary endpoint. Median levels of copeptin and hs-cTnI at admission were significantly higher in patients with an adverse outcome (Copeptin $29.6 \mathrm{pmol} / \mathrm{L}$, [IQR, 16.2-77.8] vs $17.2 \mathrm{pmol} / \mathrm{L}$ [IQR, 7.4-41.0] and hs-cTnI $22.8 \mathrm{ng} / \mathrm{L}$ [IQR, 11.5-97.5] vs $10.2 \mathrm{ng} / \mathrm{L}$ [5.5-23.1], $P<0.001$ respectively). ROC analysis demonstrated an optimal cut-off of $19.3 \mathrm{pmol} / \mathrm{L}$ for copeptin and $16.8 \mathrm{ng} / \mathrm{L}$ for hs-cTnI and an increase of either biomarker was significantly associated with the primary endpoint. The combination of raised hs-cTnI and copeptin yielded a superior prognostic value to individual measurement of biomarkers and was a strong prognostic marker upon multivariable logistic regression analysis (OR 4.274 [95\% CI, 1.995-9.154], $P<0.001$ ). Addition of copeptin and hs-cTnI to established risk models improved C-statistics and net reclassification indices.

Conclusion The combination of raised copeptin and hs-cTnI upon admission is an independent predictor of ICU admission or 28-day mortality in hospitalized patients with COVID-19.
\end{abstract}

Christoph C. Kaufmann

Christoph.C.Kaufmann@gmail.com

$1 \quad 3 R d$ Medical Department, Cardiology and Intensive Care Medicine, Klinik Ottakring (Wilhelminenhospital),

Montleartstrasse 37, 1160 Vienna, Austria

2 Department of Laboratory Medicine, Klinik Ottakring (Wilhelminenhospital), Vienna, Austria

3 Department of Hospital Hygiene, Klinik Ottakring (Wilhelminenhospital), Vienna, Austria

4 Department of Emergency Medicine, Klinik Ottakring (Wilhelminenhospital), Vienna, Austria

5 2nd Medical Department with Pneumology and Karl-Landsteiner-Institute for Lung Research and Pulmonary Oncology, Klinik Ottakring (Wilhelminenhospital), Vienna, Austria
6 Department of Gastroenterology and Hepatology, Klinik Ottakring (Wilhelminenhospital), Vienna, Austria

7 Medical School, Sigmund Freud University, Vienna, Austria

8 Department of Endocrinology and Rheumatology, Klinik Ottakring (Wilhelminenhospital), Vienna, Austria

9 Department of Internal Medicine 2, Division of Cardiology, Medical University of Vienna, Vienna, Austria

10 Core Facilities, Medical University of Vienna, Vienna, Austria

11 Ludwig Boltzmann Institute for Cardiovascular Research, Vienna, Austria

12 Medizinische Klinik III, University Hospital Heidelberg, Heidelberg, Germany 


\section{Graphical abstract}

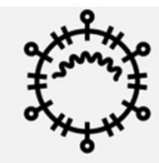

SARS-COV-2
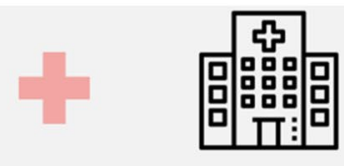

In-hospital

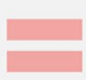

213

patients

Sample size

A 28-DAY MORTALTY ORICU ADMISSION

213 hospitalized patients with COVID-19

$55(25.8 \%)$ adverse outcome

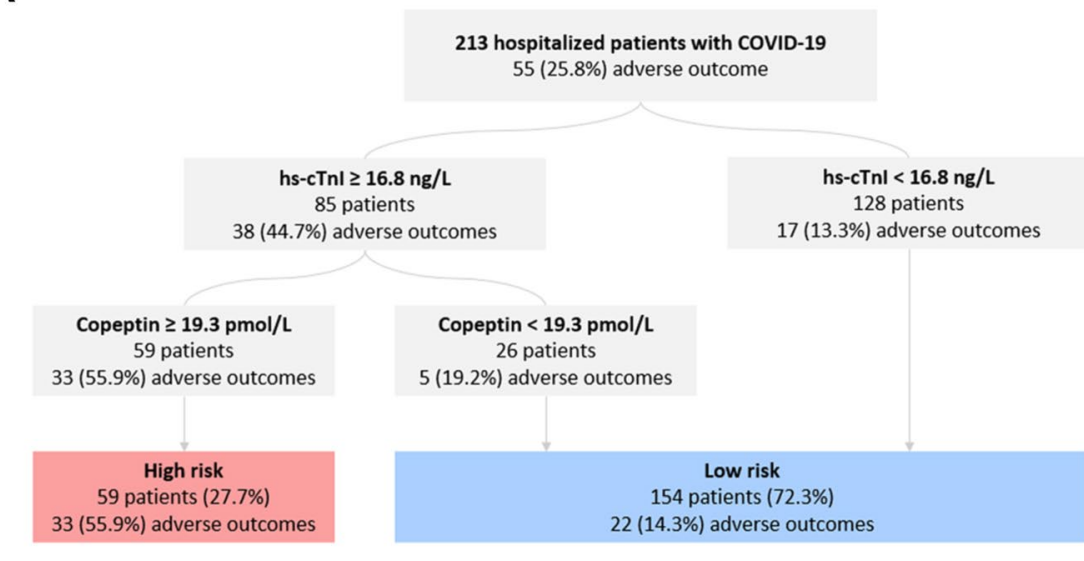

B

28-DAY MORTALITY

213 hospitalized patients with COVID-19

$28(13.1 \%)$ dead at 28 days

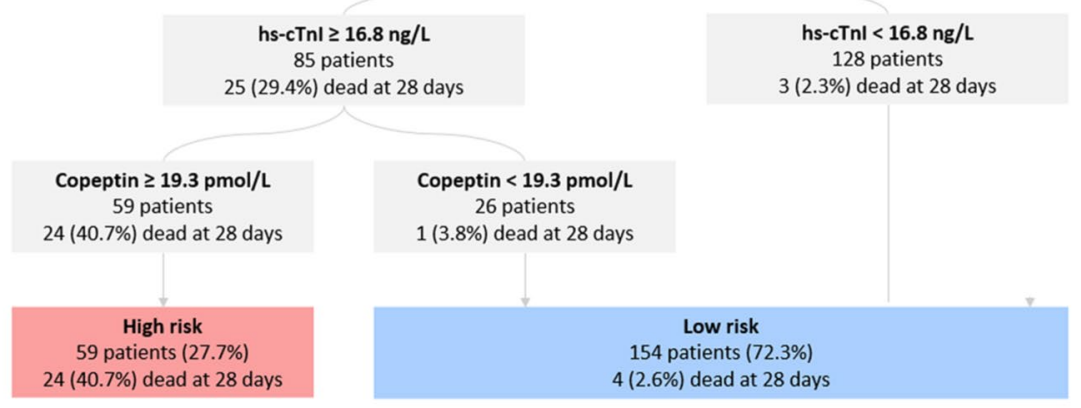

Keywords Copeptin $\cdot$ High-sensitive cardiac troponin I (hs-cTnI) $\cdot$ COVID-19 $\cdot$ Adverse outcome $\cdot$ Mortality

\section{Introduction}

Severe acute respiratory syndrome coronavirus 2 (SARSCoV-2) has been identified as the causative agent for Corona virus diasease-19 (COVID-19), which was first described late December 2019, in Wuhan, China and has since spread rapidly across the globe causing an international pandemic [1]. Identification of patients at risk is essential as high morbidity and mortality rates have been reported in patients with COVID-19, especially in those with a history of cardiovascular disease. A recent meta-analysis found a fivefold higher risk of mortality in patients with a history of cardiovascular disease [2]. Laboratory evidence of myocardial injury is a frequent finding among hospitalized patients with COVID19 and has also been established as an independent prognostic marker of adverse outcome in numerous studies [2-4]. Among patients with myocardial injury two thirds showed evidence of actual structural cardiac disease [5].

The antidiuretic hormone vasopressin plays a key role in the regulation of fluid balance and cardiovascular homeostasis and is primarily released secondary to hyperosmolarity, hypotension and stress [6]. Due to the instability of 
vasopressin in human plasma and serum, copeptin, the C-terminal fragment of the vasopressin precursor, has emerged as a surrogate marker of vasopressin as it is released in equimolar amounts [7]. Copeptin has been reported to be of prognostic value in a variety of clinical conditions, including infectious and cardiovascular disease [8-12]. Since SARSCoV-2 infection has been associated with myocardial injury, endothelial dysfunction and coagulopathy, we hypothesized that copeptin may add prognostic information in COVID19 as an additional marker of cardiovascular disease [13]. There is currently no research data available evaluating the prognostic performance of copeptin in COVID-19. Evidence from one small, retrospective study, however, suggested that raised values of copeptin facilitated differential diagnosis of patients with COVID-19 pneumonia from those with nonCOVID-19 pneumonia [14].

The aim of our study was to evaluate the performance of copeptin in combination with high-sensitive cardiac troponin I (hs-cTnI) on clinical prognosis in a well-defined cohort of hospitalized patients with COVID-19.

\section{Material and methods}

\section{Study design and patient population}

A total of 213 hospitalized patients with laboratory-confirmed SARS-CoV-2 infection and available blood samples for biomarker analysis were included in this prospective, observational, single-center study in different wards of a tertiary hospital in Vienna, Austria. All patients presented to the emergency department between June 6th to November 26th, 2020 and were admitted for in-hospital care. Patients under 18 were excluded from study participation. Prior to biomarker measurement, we excluded 10 patients with missing baseline laboratory values (e.g. hs-cTnI, $\mathrm{N}$-terminal pro-B-type natriuretic peptide [NT-proBNP] or lactate dehydrogenase [LDH]) (Supp. Figure 1). Potential SARS-CoV-2 patients were usually managed as followed at our institution: Examination was performed in a separate are of the emergency department followed by laboratory work-up, SARS-CoV-2 rapid antigen testing in the majority of patients and SARS-CoV-2 PCR in all patients. According to results of SARS-CoV-2 rapid antigen testing (available within $15 \mathrm{~min}$ ) / SARS-CoV-2 PCR patients were then admitted to a specialized SARS-CoV-2 ward. While results of SARS-CoV-2 work-up were pending, patients were continued to be cared for in the specialized area of the emergency department. The diagnosis of COVID-19 was made according to the WHO interim guidance and a polymerase chain reaction proven ribonucleic acid detection of SARS-CoV-2 on nasal and/or pharyngeal swabs was required. The study was approved by the local ethics committee of the city of Vienna (EK 20-100-VK) and complies with the Declaration of Helsinki and the International Conference on Harmonization Guidelines for Good Clinical Practice.

Demographic data, clinical features, laboratory results and medical history were obtained from patient records on admission. Preexisting cardiovascular disease was defined by a history of coronary artery disease or heart failure. Chronic pulmonary disease was defined by a history of chronic obstructive pulmonary disease, asthma bronchiale or obstructive sleep apnea. All comorbidities were defined at the discretion of the treating physician. We also analyzed electrocardiograms (ECG) upon admission and assessed the prevalence of ECG findings among patients with increased hs-cTnI and copeptin. An abnormal admission ECG was defined by the presence of atrial tachyarrhythmia (including atrial fibrillation, atrial flutter and atrial tachycardia), high degree AV block, bundle branch block, signs of left ventricular hypertrophy (Sokolow index $>3.5 \mathrm{mV}$ ), ST-segment deviation, q waves, low voltage or poor R-wave progression. The decision for admission for in-hospital care was also done at the discretion of the treating physician based on physical examination and diagnostic work-up. Follow-up data was collected through the electronic patient record system of our institution until December 19, 2020. The primary endpoint of our study was a composite of admission to the intensive care unit (ICU) or all-cause mortality within 28 days. A key secondary endpoint was 28-day all-cause mortality.

The ISARIC 4C Clinical Deterioration model was calculated for all patients as it has been shown to predict clinical deterioration (defined as any requirement of ventilatory support or critical care, or death) in patients with COVID-19 [15]. It includes age, sex, nosocomial infection, Glasgow coma scale score, peripheral oxygen saturation, breathing room air or oxygen therapy, respiratory rate, urea concentration, C-reactive protein concentration, lymphocyte count, and presence of radiographic chest infiltrates. When respiratory rate upon admission was not quantified numerically, extrapolation was made from the description of breathing patterns. Ultimately a total of 5 values for respiratory rate, 2 for radiographic chest infiltrates, 1 for peripheral oxygen saturation and 1 for blood pressure were missing, which were substituted by the median of respective values - multiple imputation and omission of missing values were performed in a second step.

We have previously published data from the same study population and reported on the prognostic impact of Midregional pro-atrial natriuretic peptide among other biomarkers (creatinine, blood urea nitrogen, LDH, hs-cTnI, NT-proBNP) on 28-day mortality [16]. 


\section{Biomarker analysis}

Serum blood samples were drawn by trained nurses or doctors upon presentation of the patient to the emergency department. After centrifugation, serum blood samples for biomarker analysis were divided into $0.5 \mathrm{ml}$ aliquots and immediately stored at $-80^{\circ} \mathrm{C}$ until measurement. Copeptin was evaluated by TRACE (Time Resolved Amplified Cryptate Emission) on the ultra-sensitive KRYPTOR compact PLUS (B.R.A.H.M.S. GmbH, Thermo Scientific, Henningsdorf, Germany). Biomarker levels were measured using commercially available kits (B.R.A.H.M.S. GmbH, Thermo Scientific, Henningsdorf, Germany) according to the manufacturer's guidelines. According to the manufacture's specification, the detection limit of copeptin was set at $0.70 \mathrm{pmol} / \mathrm{L}$ and the upper limit at $2000 \mathrm{pmol} / \mathrm{L}$. In patients with suspected acute coronary syndrome, a copeptin value $<95$ th percentile upper limit of normal (ULN), i.e. $<10 \mathrm{pmol} / \mathrm{L}$ together with a cTn or hs-cTn $<99$ th percentile ULN was used for instant rule out of MI. High-sensitive cardiac troponin I was measured using the Dimension Vista High-sensitivity Troponin I Assay (Siemens Healthcare Diagnostics), which is based on a luminescent oxygen channeling immunoassay. According to the manufacture's specification, the detection limit of hs-cTnI was set at $3.0 \mathrm{ng} / \mathrm{L}$ and the upper limit at $125000 \mathrm{ng} / \mathrm{L}$ [17].

Copeptin was measured as part of the research protocol months after admission of the patients and the treating physicians were therefore blinded to the results, while hs-cTnI was measured during clinical routine-hence being available to the treating physician. Further routine assessment of laboratory values was performed at the certified central laboratory of the Wilhelminenhospital and included: white blood cells, neutrophil granulocytes, lymphocytes, c-reactive protein (CRP), platelets, hemoglobin, creatinine, sodium, potassium, and $\mathrm{LDH}$, respectively. Neutrophil-to-lymphocyte ratio (NLR) was calculated by dividing the absolute neutrophil count by the absolute lymphocyte count.

We also assessed turn-around times for biomarkers and admission protocols to give further insight into the timing of events of our study:

- Turn-around times for hs-cTnI: $151 \mathrm{~min}$ (IQR, 90-360).

- Turn-around times for SARS-CoV-2 PCR: $193 \mathrm{~min}$ (IQR, 128-352).

- Time from ED registration to hs-cTnI blood drawing: 56 min (IQR, 38-93).

- Time from ED registration to copeptin blood drawing: 54 min (IQR, 38-88).

- Time from ED registration to SARS-CoV-2 nasopharyngeal swab: $69 \min$ (IQR, 44-120).

- Time from ED registration to registration on COVID-19 ward: 244 min (IQR, 195-306).

\section{Statistical analysis}

Continuous data are reported as median and interquartile range (IQR), and categorical data are expressed as frequency and percentage. Normality was checked using the Shapiro-Wilk normality test. Mann-Whitney $U$-test and Pearson's chi-squared test were used to compare continuous and categorical data between patients with an adverse outcome and those without an adverse outcome.

Youden-index quantification was used to identify the optimal cut-off values of biomarkers for prediction of the primary endpoint. We calculated specificity, sensitivity, negative and positive predictive values and the negative and positive likelihood ratios for all biomarkers. Comparison of event rates across different risk classes was performed using Pearson's chi-squared test. Univariable binary logistic regression analysis was performed to determine the prognostic impact of baseline characteristics, comorbidities, clinical symptoms and laboratory markers. The independence of the prognostic value of copeptin and hs-cTnI was tested by adjusting for age and gender (model 1 ), model $1+$ comorbidities and clinical symptoms (model 2 [baseline model]). Variables found to be significant upon univariable regression analysis were included in a final multivariable model using the backward stepwise selection method. We also calculated the C-statistics, integrated discrimination improvement index (IDI), and category-free net reclassification improvement index (NRI) to evaluate the discriminatory properties of copeptin and hs-cTnI in addition to established risk models (baseline model and ISARIC 4C deterioration model).

All statistical analyses were performed using SPSS 26.0 (SPSS Inc., Chicago, IL, USA) and RStudio (v 1.0.143). Graphics were generated using GraphPad Prism 9.0 (GraphPad Software, Inc., San Diego, CA). A two-sided $p$-value $<0.05$ was required for statistical significance.

\section{Results}

\section{Clinical characteristics and biomarkers stratified by the primary endpoint}

A total of 213 hospitalized patients with COVID-19 were included in the study. Mean age was 65.6 years $(\mathrm{SD} \pm 16.8$ years $)$ and $119(55.9 \%)$ were male. Within 28 days of admission, 55 patients $(25.8 \%)$ reached the primary endpoint of ICU admission or 28-day mortality and 28 patients (13.2\%) reached the secondary endpoint of 28-day mortality. Patients with an unfavorable outcome were significantly older, more frequently male, and more often had a history of hypertension, cardiovascular disease and/or chronic kidney disease. While dyspnea was more common in patients reaching the primary endpoint, no difference was 
observed with regards to diabetes mellitus, chronic pulmonary disease or fever and coughing (Table 1).

Median levels of both copeptin and hs-cTnI at admission were significantly higher in patients with the primary endpoint as compared to those with a favorable disease course (Copeptin: $29.6 \mathrm{pmol} / \mathrm{L}$ IQR, [IQR, 16.2-77.8] vs $17.2 \mathrm{pmol} / \mathrm{L}$ [IQR, 7.4-41.0], $P<0.001$; hs-cTnI: $22.8 \mathrm{ng} / \mathrm{ml}$ [IQR, 11.5-97.5] vs $10.2 \mathrm{ng} / \mathrm{ml}$ [IQR, 5.5-23.1], $P<0.001$ ). Of other laboratory markers, white blood cells, neutrophil granulocytes, CRP, creatinine, NLR and LDH were significantly higher in those with subsequent ICU admission or death, while lymphocytes were lower. A second hs-cTnI measurement within $36 \mathrm{~h}$ was available in 53 of 213 patients with concentrations of hs-cTnI being higher upon admission (1 st hs-cTnI: 16.7 [IQR, 7.0-66.1], 2nd hs-cTnI: 13.3 [IQR, 6.2-50.5]; $P=0.016$ ). (Table 1 and Fig. 1).

\section{Correlation of copeptin with other markers, relation to time to symptom onset and ECG changes}

A weak to moderate correlation of copeptin levels was observed with markers of inflammation (CRP: $r=0.160$;
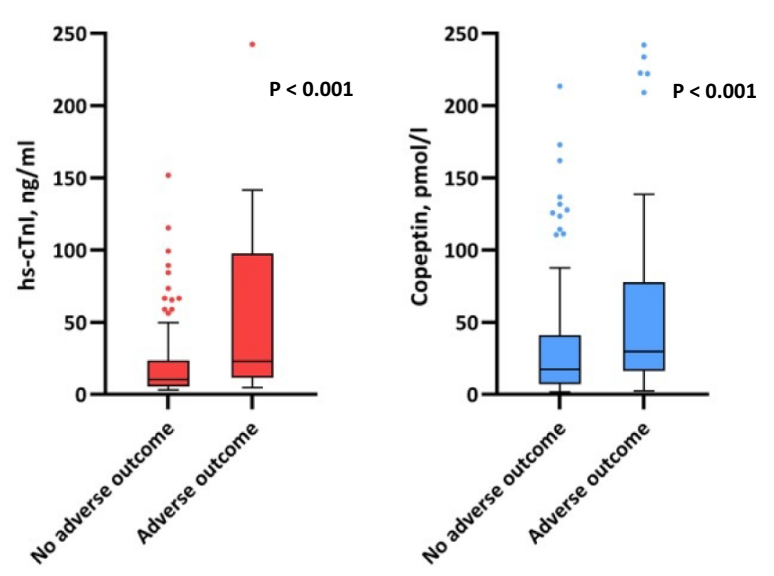

Fig. 1 Box plots of hs-cTnI and Copeptin levels stratified by the primary endpoint

$P=0.020$ and NLR: $r=0.392, P<0.001)$ renal failure (Creatinine: $r=0.420 ; P<0.001$ ), myocardial injury (hs-cTnI: $r=0.440 ; P<0.001)$ and age $(r=0.513, P<0.001)$. Patients with a history of hypertension, cardiovascular disease or
Table 1 Baseline characteristics of the study population stratified by primary study end point

\begin{tabular}{|c|c|c|c|}
\hline Characteristics & Non-ICU survivor $(n=158)$ & ICU or death $(n=55)$ & $P$-value \\
\hline \multicolumn{4}{|l|}{ Baseline characteristics } \\
\hline Age, years & $63.5 \pm 16.46$ & $72.0 \pm 16.25$ & 0.001 \\
\hline Male sex & $82(51.9 \%)$ & $37(67.3 \%)$ & 0.048 \\
\hline Arterial hypertension & $84(53.2 \%)$ & $38(69.1 \%)$ & 0.040 \\
\hline Diabetes mellitus & $46(29.1 \%)$ & $21(38.2 \%)$ & 0.213 \\
\hline Cardiovascular disease & $23(14.6 \%)$ & $21(38.2 \%)$ & $<0.001$ \\
\hline Chronic pulmonary disease & $20(12.7 \%)$ & $8(14.5 \%)$ & 0.722 \\
\hline Chronic kidney disease & $21(13.3 \%)$ & $15(27.3 \%)$ & 0.017 \\
\hline History of malignancy & $14(8.9 \%)$ & $9(16.4 \%)$ & 0.123 \\
\hline \multicolumn{4}{|l|}{ Signs and symptoms } \\
\hline Fever & $127(80.4 \%)$ & $38(69.1 \%)$ & 0.084 \\
\hline Coughing & $89(56.3 \%)$ & $25(45.5 \%)$ & 0.164 \\
\hline Dyspnea & $87(55.1 \%)$ & $42(76.4 \%)$ & 0.005 \\
\hline \multicolumn{4}{|l|}{ Laboratory values at admission } \\
\hline White blood cells, G/1 & $6.5(5.2-8.5)$ & $7.8(5.8-10.3)$ & 0.039 \\
\hline Neutrophil granulocytes, G/l & $4.9(3.6-6.5)$ & $6.2(4.1-8.8)$ & 0.003 \\
\hline Lymphocytes, G/l & $1.08(0.77-1.60)$ & $0.77(0.57-1.03)$ & $<0.001$ \\
\hline Neutrophil to lymphocyte ratio & $4.1(2.5-7.6)$ & $8.1(5.9-11.9)$ & $<0.001$ \\
\hline C-reactive protein, $\mathrm{mg} / \mathrm{L}$ & $58(25-110)$ & $90(53-172)$ & $<0.001$ \\
\hline Hemoglobin, g/dL & $13.6(12.3-14.6)$ & $13.3(12.0-14.6)$ & 0.417 \\
\hline Platelets, G/L & $199(160-241)$ & $206(169-253)$ & 0.492 \\
\hline Creatinine, $\mathrm{mg} / \mathrm{dL}$ & $1.0(0.8-1.2)$ & $1.2(0.9-1.6)$ & $<0.001$ \\
\hline Sodium, mmol/L & $137(135-139)$ & $137(134-138)$ & 0.833 \\
\hline Potassium, mmol/L & $4.0(3.8-4.2)$ & $4.0(3.7-4.2)$ & 0.317 \\
\hline Lactate dehydrogenase, U/L & $276(222-354)$ & $326(239-500)$ & 0.007 \\
\hline Copeptin, pmol/L & $17.2(7.4-41.0)$ & $29.6(16.2-77.8)$ & $<0.001$ \\
\hline Hs-cTnI, ng/L & $10.2(5.5-23.1)$ & $22.8(11.5-97.5)$ & $<0.001$ \\
\hline
\end{tabular}




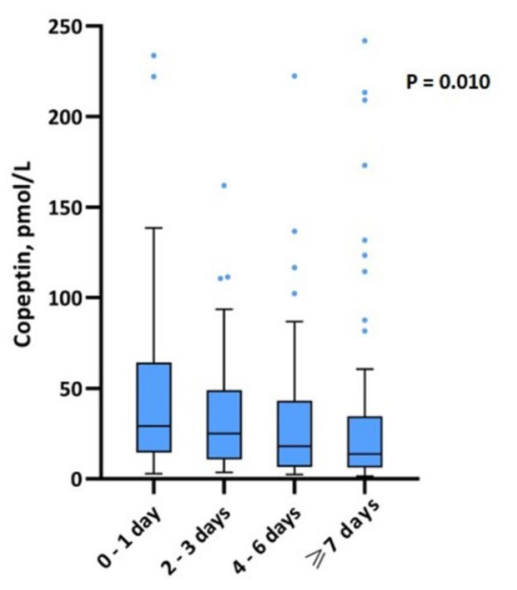

Fig. 2 Copeptin levels stratified by quartiles of symptom onset

chronic kidney disease had significantly higher levels of copeptin upon admission ( $p<0.001$, respectively).

We also found significantly increased values of copeptin among those presenting early in the course of disease, which was assessed by time to onset of symptoms ( $0-1$ days: Copeptin $39.1 \mathrm{pmol} / \mathrm{L}$ [IQR, 14.9-63.6] vs. $>7$ days: Copeptin $13.7 \mathrm{pmol} / \mathrm{L}$ [IQR, 6.3-33.9]; $P=0.014$ ) (Fig. 2).

Patients with increased hs-cTnI and copeptin had a significantly higher prevalence of an abnormal admission ECG compared to those without increased hs-cTnI and copeptin (78\% vs $45 \% ; P$ - Value $<0.001$ ). With regards to specific ECG changes, we observed a significantly higher rate of atrial tachyarrhythmia, bundle branch block, low voltage and poor R-wave progression among those with increased hs-cTnI and copeptin (Fig. 3).

\section{Prognostic value of copeptin and hs-cTnl for prediction of 28-day adverse outcomes}

A copeptin level of $19.3 \mathrm{pmol} / \mathrm{L}$ and a hs-cTnI level of $16.8 \mathrm{ng} / \mathrm{L}$ were identified as the optimal cut-off levels for the prediction of the primary endpoint according to Youden's index. While the prognostic specificity of hs-cTnI was higher compared to copeptin (70.3\% vs $56.3 \%$ ), sensitivity was comparable (70.9\% with copeptin vs $69.1 \%$ with hscTnI) with the combination of copeptin / hs-cTnI yielding the highest specificity of $83.5 \%$ (Table 2).

Upon univariable logistic regression both elevated copeptin and hs-cTnI (according to the threshold identified by ROC analysis) were significant predictors of an adverse outcome within 28 days of hospital admission (Copeptin: crude OR 3.144 [95\% CI 1.623-6.091]; $P=0.001$ and hscTnI: crude OR 5.279 [95\% CI 2.713-10.274]; $P<0.001$ ). This association remained statistically significant for both biomarkers after adjustment for age and gender (model 1) as well as comorbidity burden and clinical symptoms (model
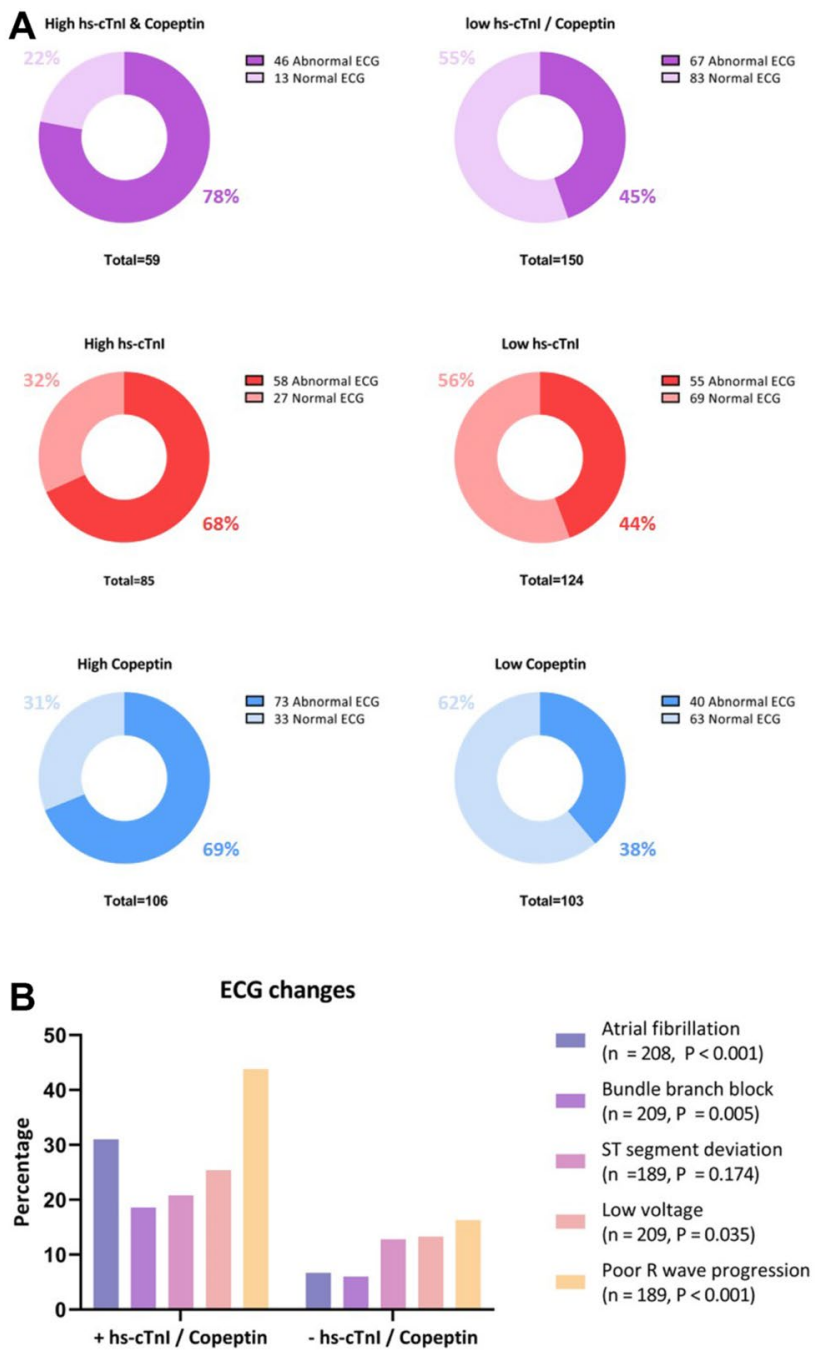

Fig. 3 A Prevalence of abnormal ECG among patients with high hs-cTnI and copeptin, patients with high hs-cTnI only and patients with high copeptin only B ECG changes among patients with high hs-cTnI and copeptin compared to those without increased hs-cTnI and copeptin. Abnormal ECG was defined by the presence of one (or more) of the following: atrial tachyarrhythmia / high degree AV block, bundle branch block, ST-segment deviation, Q waves, left ventricular hypertrophy, low voltage and poor R-wave progression

2). Results were consistent when using biomarkers on a categorical or continuous level. (Table 3 and Supp. Table 3) A final regression model with backwards selection of variables, including baseline characteristics, comorbidities, clinical symptoms as well as other established laboratory markers of risk in COVID-19, revealed that the combination of raised hs-cTnI and copeptin was associated with a fourfold greater risk of an unfavorable outcome (OR 4.274 [95\% CI 1.995-9.154]; $P<0.001)$. Other factors found to be significantly associated with the primary endpoint were dyspnea, raised creatinine and neutrophil to lymphocyte ratio. (Supp. Table 1). We also observed a significant association of the 
Table 2 Sensitivity, specificity, negative predictive value and positive predictive value of Copeptin, hs-cTnI and Copeptin / hs-cTnI for the primary endpoint

\begin{tabular}{lllllll}
\hline Laboratory biomarker & Sensitivity & Specificity & LR + & LR - & PPV & NPV \\
\hline Copeptin $\geq 19.3 \mathrm{pmol} / \mathrm{L}$ & $70.9 \%$ & $56.3 \%$ & 1.62 & 0.52 & $36.1 \%$ & $84.8 \%$ \\
$\mathrm{Hs}-\mathrm{cTnI} \geq 16.8 \mathrm{ng} / \mathrm{L}$ & $69.1 \%$ & $70.3 \%$ & 2.33 & 0.44 & $44.7 \%$ & $86.7 \%$ \\
$\begin{array}{l}\text { Copeptin } \geq 19.3 \mathrm{pmol} / \mathrm{L} \text { and } \\
\text { hs-cTnI } \geq 16.8 \mathrm{ng} / \mathrm{L}\end{array}$ & $60.0 \%$ & $83.5 \%$ & 3.64 & 0.48 & $55.9 \%$ & $85.7 \%$ \\
\hline
\end{tabular}

$N P V$ negative predictive value, $P P V$ positive predictive value

\begin{tabular}{|c|c|c|c|c|c|c|c|c|c|}
\hline \multirow[t]{2}{*}{ Statistical model } & \multicolumn{3}{|c|}{ Copeptin $>19.3 \mathrm{pmol} / \mathrm{L}$} & \multicolumn{3}{|c|}{ Hs-cTnI > $16.8 \mathrm{ng} / \mathrm{L}$} & \multicolumn{3}{|c|}{$\begin{array}{l}\text { Hs-cTnI }>16.8 \mathrm{ng} / \mathrm{L} \text { and } \\
\text { Copeptin }>19.3 \mathrm{pmol} / \mathrm{L}\end{array}$} \\
\hline & OR & $95 \% \mathrm{CI}$ & $P$-value & OR & $95 \% \mathrm{CI}$ & $P$-value & OR & $95 \% \mathrm{CI}$ & $P$-value \\
\hline Crude model & 3.14 & $1.62-6.09$ & 0.001 & 5.28 & $2.71-10.27$ & $<0.001$ & 7.62 & $3.84-15.10$ & $<0.001$ \\
\hline Model $1^{\mathrm{a}}$ & 2.23 & $1.06-4.70$ & 0.034 & 3.84 & $1.79-8.24$ & 0.001 & 6.66 & $2.93-15.12$ & $<0.001$ \\
\hline Model $2^{\mathrm{b}}$ & 2.45 & $1.14-5.27$ & 0.022 & 3.22 & $1.44-7.20$ & 0.004 & 6.02 & $2.55-14.19$ & $<0.001$ \\
\hline
\end{tabular}

The crude model only included the biomarker in question; Model 1 was adjusted for age and gender; Model 2 was adjusted for Model 1 and arterial hypertension, history of cardiovascular disease, chronic kidney disease and dyspnea
Table 3 Association of Copeptin, hs-cTnI and Copeptin / hs-cTnI with the primary endpoint model with the combination of both biomarkers providing the best prognostic information. These findings were underlined by a statistically significant risk reclassification for copeptin, hs-cTnI and combined copeptin / hs-cTnI upon addition to both risk models. The combination of copeptin with hs-cTnI was also superior to the combination of MRproANP with hs-cTnI in terms of predictive performance in our study population. The category-free NRI for combined copeptin/hs-cTnI was 0.653 (95\% CI 0.360-0.945, $P<0.001)$ and 0.098 (95\% CI $0.056-0.140, P<0.001)$ and the IDI was $0.871(95 \%$ CI $0.587-1.155, P<0.001)$ and 0.072 (95\%CI $0.030-0.114, P<0.001)$ when added to the baseline risk model and the ISARIC 4C Clinical deterioration model, respectively. We used different methods to account for the small number of missing values (substitution of missing values by the median, omission of missing values and multiple imputation of missing values) and found a consistent pattern of statistical performance for all biomarkers (Supp. Table 4).

\section{Discussion}

This prospective, observational single-center study shows for the first time the prognostic impact of copeptin in a wellcharacterized cohort of hospitalized patients with COVID19. The main findings are (i) that levels of copeptin and hs-cTnI upon hospital admission were significantly higher among those with a subsequent adverse course of disease; (ii) an association of increased copeptin and hs-cTnI with the primary endpoint, both individually and in combination, which persisted after multivariable adjustment; (iii) a 
Fig. 4 Risk assessment strategy based on hs-cTnI and levels of copeptin in patients with COVID-19
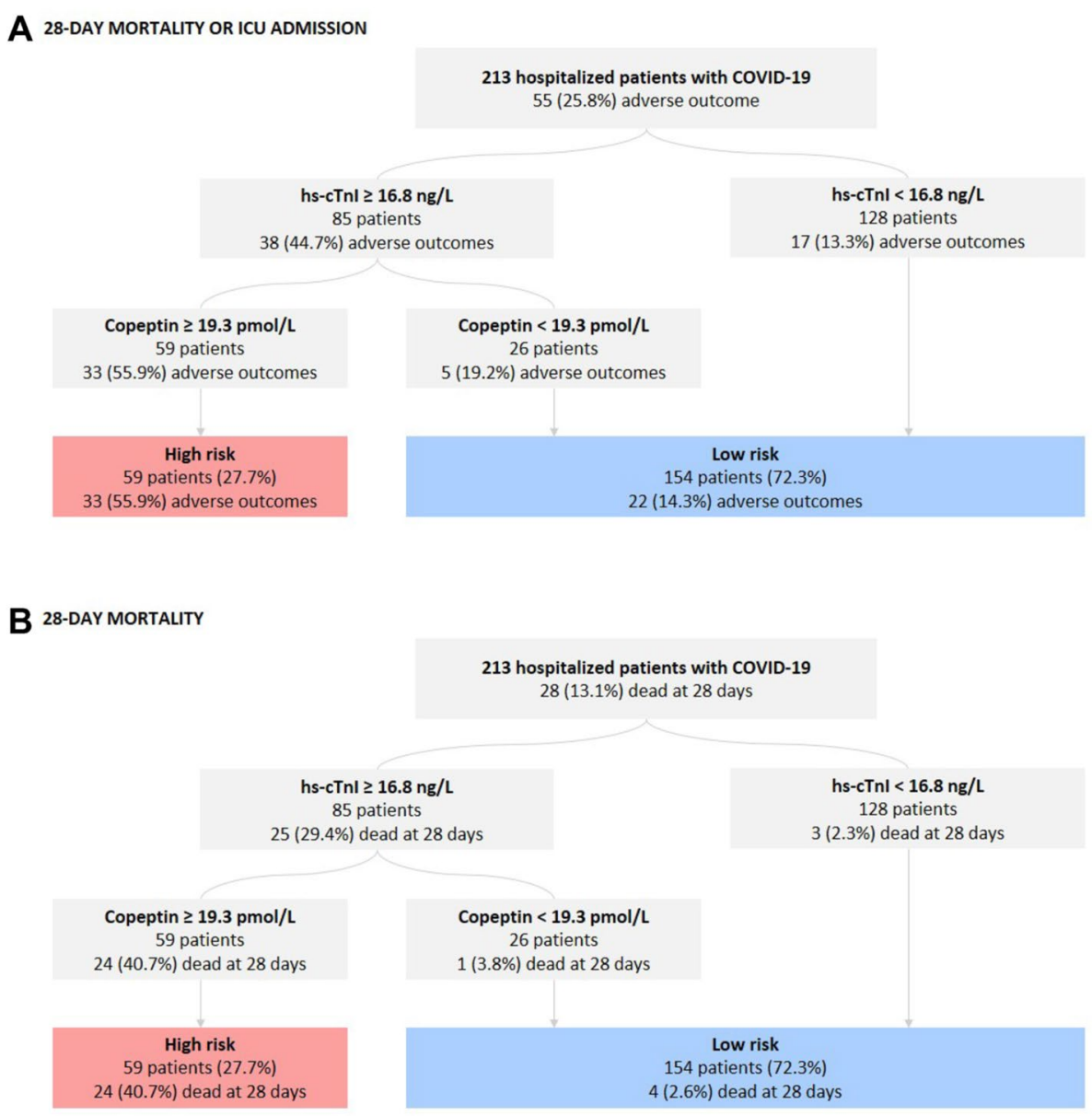

213 hospitalized patients with COVID-19 $28(13.1 \%)$ dead at 28 days
hs-cTnl $\geq 16.8 \mathrm{ng} / \mathrm{L}$ 85 patients

(29.4\%) dead at 28 days
hs-cTnl $<16.8 \mathrm{ng} / \mathrm{L}$ 128 patients $3(2.3 \%)$ dead at 28 days significant risk reclassification of patients with increased hscTnI by the addition of copeptin with regards to both study endpoints; and (iv) an incremental value of the combination of raised copeptin and hs-cTnI for outcome prediction when added to the baseline risk model of our study or the ISARIC 4C Clinical deterioration model.

The antidiuretic hormone vasopressin plays a key role in the regulation of fluid balance and cardiovascular homeostasis. The most powerful stimulus for vasopressin release is a change in plasma osmolality with even small changes being of clinical significance. Vasopressin has also been shown to increase in response to stress or acute-life threating conditions as it influences the body's response to both acute and chronic stressful conditions [6]. Copeptin, the c-terminal fragment of the vasopressin precursor protein, is characterized by excellent ex vivo stability as it is cleared less rapidly from plasma compared to vasopressin, which makes it a viable surrogate marker of vasopressin release, that can be used for routine measurement in clinical practice [7]. Our data show that copeptin levels were increased in hospitalized patients with COVID-19, as the median levels (19.5 pmol/L, IQR 8.9-46.7) were above the established normal values of a healthy population (median of $4.2 \mathrm{pmol} / \mathrm{L}$,
IQR 1-13.8 pmol/L). To date, only one study has published data on copeptin measurements in patients with COVID-19 pneumonia $(n=98)$ and found significantly increased values compared to patients with non-COVID-19 pneumonia [14].

Although the underlying mechanism by which copeptin is linked to COVID-19 has not been elucidated yet, several potential mechanisms may play a role in the observed association. First, we should consider that raised levels of copeptin have been reported in several clinical conditions and are not specific to a certain situation or organ manifestation. Similarly to our findings, higher levels of copeptin have been reported in patients with arterial hypertension, diabetes mellitus, chronic kidney disease and history of cardiovascular disease $[11,12,18]$. Thus, the increase of copeptin may be partially explained by a higher baseline comorbidity burden. This is in line with our findings of a higher rate of abnormal admission ECGs among patients with increased hs-cTnI and copeptin, which may reflect a history of cardiovascular disease or cardiovascular morbidity in general. Another potential pathophysiological link may lie in the association of copeptin with infectious diseases and inflammation [19]. We also found a significant correlation of copeptin with CRP and NLR, as surrogate markers 
Table 4 Reclassification and discrimination statistics (95\% CIs) for the primary endpoint

\begin{tabular}{|c|c|c|c|c|c|}
\hline Clinical risk model & C-statistic $(95 \% \mathrm{CI})$ & NRI $(95 \% \mathrm{CI})$ & $P$-value & IDI $(95 \% \mathrm{CI})$ & $P$-value \\
\hline Copeptin & $\begin{array}{c}0.636 \\
(0.564-0.708)\end{array}$ & & & & \\
\hline MR-proANP & $\begin{array}{c}0.667 \\
(0.597-0.738)\end{array}$ & & & & \\
\hline Hs-cTnI & $\begin{array}{c}0.697 \\
(0.626-0.768)\end{array}$ & & & & \\
\hline MR-proANP / hs-cTnI & $\begin{array}{l}0.695 \\
(0.623-0.768)\end{array}$ & & & & \\
\hline Copeptin / hs-cTnI & $\begin{array}{l}0.718 \\
(0.646-0.789)\end{array}$ & & & & \\
\hline Baseline model & $\begin{array}{c}0.802 \\
(0.735-0.870)\end{array}$ & & & & \\
\hline Baseline model + Copeptin & $\begin{array}{c}0.816 \\
(0.747-0.886)\end{array}$ & $\begin{array}{l}0.545 \\
(0.259-0.830)\end{array}$ & $<0.001$ & $\begin{array}{r}0.026 \\
(0.004-0.048)\end{array}$ & 0.019 \\
\hline Baseline model + MR-proANP & $\begin{array}{c}0.808 \\
(0.739-0.877)\end{array}$ & $\begin{array}{l}0.377 \\
(0.076-0.678)\end{array}$ & 0.014 & $\begin{array}{r}0.027 \\
(0.005-0.049)\end{array}$ & 0.015 \\
\hline Baseline model + hs-cTnI & $\begin{array}{c}0.815 \\
(0.751-0.879)\end{array}$ & $\begin{array}{l}0.634 \\
(0.3491-0.918)\end{array}$ & $<0.001$ & $\begin{array}{r}0.025 \\
(0.001-0.050)\end{array}$ & 0.046 \\
\hline Baseline model + Copeptin / hs-cTnI & $\begin{array}{l}0.829 \\
(0.765-0.893)\end{array}$ & $\begin{array}{l}0.702 \\
(0.412-0.991)\end{array}$ & $<0.001$ & $\begin{array}{r}0.072 \\
(0.032-0.112)\end{array}$ & $<0.001$ \\
\hline Baseline model + MR-proANP / hs-cTnI & $\begin{array}{l}0.810 \\
(0.743-0.877)\end{array}$ & $\begin{array}{l}0.499 \\
(0.205-0.793)\end{array}$ & $<0.001$ & $\begin{array}{r}0.027 \\
(0.003-0.051)\end{array}$ & 0.03 \\
\hline $4 \mathrm{C}$ deterioration model & $\begin{array}{c}0.799 \\
(0.731-0.867)\end{array}$ & & & & \\
\hline 4C deterioration model + Copeptin & $\begin{array}{l}0.804 \\
(0.736-0.871)\end{array}$ & $\begin{array}{l}0.545 \\
(0.259-0.830)\end{array}$ & $<0.001$ & $\begin{array}{c}0.014 \\
(-0.004-0.031)\end{array}$ & 0.126 \\
\hline $4 \mathrm{C}$ deterioration model $+\mathrm{MR}$-proANP & $\begin{array}{l}0.806 \\
(0.737-0.876)\end{array}$ & $\begin{array}{l}0.670 \\
(0.389-0.950)\end{array}$ & $<0.001$ & $\begin{array}{r}0.024 \\
(0.004-0.045)\end{array}$ & 0.021 \\
\hline $4 \mathrm{C}$ deterioration model $+\mathrm{hs}-\mathrm{cTnI}$ & $\begin{array}{l}0.818 \\
(0.753-0.884)\end{array}$ & $\begin{array}{l}0.787 \\
(0.504-1.070)\end{array}$ & $<0.001$ & $\begin{array}{r}0.037 \\
(0.009-0.006)\end{array}$ & 0.009 \\
\hline 4C deterioration model + Copeptin / hs-cTnI & $\begin{array}{c}0.837 \\
(0.774-0.900)\end{array}$ & $\begin{array}{l}0.871 \\
(0.587-1.155)\end{array}$ & $<0.001$ & $\begin{array}{r}0.072 \\
(0.030-0.114)\end{array}$ & $<0.001$ \\
\hline $4 \mathrm{C}$ deterioration model $+\mathrm{MR}$-proANP / hs-cTnI & $\begin{array}{c}0.816 \\
(0.748-0.883)\end{array}$ & $\begin{array}{l}0.781 \\
(0.493-1.069)\end{array}$ & $<0.001$ & $\begin{array}{r}0.039 \\
(0.011-0.067)\end{array}$ & $<0.001$ \\
\hline
\end{tabular}

The base model included age, gender, arterial hypertension, history of cardiovascular disease, chronic kidney disease, dyspnea, heart rate, respiratory rate, mean blood pressure and body temperature

of inflammation, which have both been reported to be of prognostic value in COVID-19 [20, 21]. Copeptin has also emerged as a promising biomarker of the non-specific stress response to acute illness, which may further contribute to risk stratification in COVID-19 as SARS-CoV-2 infection can trigger a substantial stress response in some individuals [22]. Furthermore, Lin et al. have previously demonstrated that increased values of copeptin were highly specific for diagnosing acute respiratory distress syndrome (ARDS) and acute lung injury [23]. Since ARDS is a critical complication of COVID-19, copeptin levels upon admission may thus facilitate identification of patients at risk for ARDS and a subsequent fatal clinical course.

Regardless of the underlying pathophysiology, our study findings corroborate the role of copeptin to predict adverse outcomes in a variety of clinical conditions by extending its use to patients with COVID-19. In our study, the optimal cut-off point of copeptin to predict the primary endpoint was $19.3 \mathrm{pmol} / \mathrm{L}$, which is above the 99 th percentile of $13.5 \mathrm{pmol} / \mathrm{L}$ found in a healthy population and the suggested cut-off of $10 \mathrm{pmol} / \mathrm{L}$ for the rule-out of myocardial infarction. Interestingly, the optimal cut-off of hs-cTnI (16.8 ng/L) to predict the primary endpoint was well below the 99th percentile of $59 \mathrm{ng} / \mathrm{L}$.

We also show that using a dual marker strategy by measuring copeptin and hs-cTnI together upon hospital admission for acute SARS-CoV-2 infection adds significant prognostic value to established risk prediction models for COVID-19. An incremental discriminatory and reclassification predictive value of copeptin, hs-cTnI and the combination of copeptin / hs-cTnI upon addition to the baseline model of our study and the ISARIC 4C clinical deterioration 
model - which has been validated as a potent risk score in COVID-19-was observed [15]. The highest C-statistics, NRI and IDI values were recorded for the combination of raised copeptin and hs-cTnI. Only patients with both increased hs-cTnI and copeptin had a markedly higher risk of an adverse outcome, while patients with raised hs-cTnI and copeptin levels below the optimal cut-off point had a similar prognosis to those with low values of hs-cTnI. A similar risk reclassification was also observed for the secondary endpoint of 28-day mortality. Our proposed dual biomarker strategy may help to identify patients at risk of an adverse outcome and hence guide early management. The combination of copeptin and hs-cTnI provides significant, additional prognostic value over the single use of hs-cTnI, as evidenced by the findings of our study. However, we should also consider that the $\mathrm{C}$-statistics of 0.718 for the combination of increased hs-cTnI and copeptin is rather modest and hence has to be interpreted within the context of the individual clinical situation. This observation in patients with confirmed COVID-19 is very consistent with findings in 3890 patients presenting with acute symptoms to the emergency department [24]. In a multivariate Cox regression model, elevated copeptin was independently associated with all-cause death in the acute coronary syndrome $(\mathrm{HR}=1.7$ (95\% CI 1.3-2.3), $p=0.002$ ) and non-acute coronary syndrome cohort $(\mathrm{HR}=2.7$ (95\% CI 1.4-5.0), $p=0.0018)$. Among patients with elevated hs-cTnT, mortality rates were almost three-times higher in patients with increased compared to those with normal copeptin levels $(29.0 \%$ vs. $10.7 \%$, $p<0.001)$. Taking these results into account we propose a simple risk stratification model based on admission levels of hs-cTnI and copeptin with the high-risk group constituting patients with an increase of both biomarkers and the low-risk group constituting the rest of patients. A similar biomarker guided model has been suggested for risk stratification in patients with normotensive pulmonary embolism, which is based on the measurement of cardiac troponin, Nt-proBNP and copeptin [25]. Other authors have also underlined the diagnostic and prognostic value of a dual marker strategy by measuring both copeptin and high-sensitive cardiac troponin in patients with suspected myocardial infarction [26-32]. The clinical relevance of cardiovascular biomarker testing in patients with COVID-19 has been discussed extensively in recent months. A recently published paper by the ESC Study Group on Biomarkers in Cardiology of the Acute Cardiovascular Care Association underlined the prognostic information gained by cardiovascular biomarker measurement-mainly cardiac troponin, natriuretic peptides and d-dimer-which in conjunction with vital parameters may help treating physicians to identify patients at risk of deterioration. Our study proposes an improvement of outcome prediction by the addition of copeptin to hs-cTnI, which may ultimately help decision making in the emergency department [33].

This prospective single-center study has several limitations that require further discussion. Since only patients admitted for in-hospital treatment were included, no extrapolation can be made to patients being discharged from the emergency department. The decision for hospital admission was made by the treating physician based on clinical examination and diagnostic work-up. Second, before recommending the combination of increased copeptin and hs-cTnI as a robust predictor of worse outcome in COVID-19, it should be validated in an independent population. Third, we used baseline levels of copeptin and hs-cTnI and hence cannot draw conclusions as to how temporal changes of both biomarkers may affect the outcome. Fourth, a small number of patients had missing values for the calculation of the ISARIC 4C clinical deterioration score (as specified above), which were substituted by the median values of respective parameters alongside confirming our results by multiple imputation and by omitting patients with missing values. However, since only a couple of data points were missing this should not have a significant effect on our findings. Fifth, since this is a single-center study external validation of our results has to be done before recommendations for routine clinical practice can be made. We also have to consider that only hs-cTnI assays were used in our study and we cannot reliably extrapolate our findings to other troponin assays (e.g. commonly used hs-cTnT assay). Reported turn-times of hscTnI and SARS-CoV-PCR should be considered with the knowledge that preliminary results before final validation by a laboratory physician can be accessed by the treating physician and may have an impact on decision made in the emergency department. Measurement of copeptin levels also depends on the availability of appropriate laboratory equipment, which may limit its use in some hospitals. Finally, we only report short-term event rates in our study population and therefore cannot assess the prognostic impact on longterm outcomes.

\section{Conclusions}

In our study population of hospitalized patient with COVID19, copeptin refined the prognostic impact of hs-cTnI by reclassifying patients to low or high risk. The combination of increased copeptin and hs-cTnI independently predicted the primary endpoint of 28-day mortality or ICU admission and was of incremental prognostic value upon addition to established risk models for COVID-19 and the baseline model of our study. Our study findings suggest a role of copeptin in addition to hs-cTnI as dual marker strategy for early risk stratification in patients with COVID-19. 
Supplementary Information The online version contains supplementary material available at https://doi.org/10.1007/s00392-021-01970-4.

Author contributions All authors contributed to the manuscript and read and approved the final version of the manuscript.

Funding This work was supported by the "Bürgermeisterfond der Stadt Wien", the Association for the Promotion of Research on Arteriosclerosis, Thrombosis, and Vascular Biology (ATVB) and by the Ludwig Boltzmann Cluster for Cardiovascular Research.

Availability of data and material The datasets generated for this study are available on request to the corresponding author.

\section{Declarations}

Conflicts of interest Dr. Huber reports Honoraria for lectures from AstraZeneca, Bayer, Boehringer Ingelheim, B.R.A.H.M.S Thermo Fisher, Daiichi Sankyo, Pfizer, Sanofi and The Medicines Company and has received research funding form AstraZeneca and B.R.A.H.M.S Thermo Fisher. Dr. Giannitsis reports Honoraria for lecturers from Roche Diagnostics, BRAHMS Thermo Fisher Scientific, Bayer Vital $\mathrm{GmbH}$ and Mitsubishi Chemical Europe, institutional research grant from Roche Diagnostics and Daiichi Sankyo, consultant for Roche Diagnostics and BRAHMS Thermo Fisher Scientific, outside the submitted work. The other authors do not have any conflicts of interest to disclose.

Ethics approval Research involving human subjects complied with all relevant national regulations, institutional policies and is in accordance with the tenets of the Helsinki Declaration (as revised in 2013) and has been approved by the local ethics committee of the city of Vienna (EK 20-100-VK).

\section{References}

1. Fauci AS, Lane HC, Redfield RR (2020) Covid-19 - navigating the uncharted. N Engl J Med 382:1268-1269

2. Li X, Guan B, Su T, Liu W, Chen M, Bin Waleed K, Guan X, Gary T, Zhu Z (2020) Impact of cardiovascular disease and cardiac injury on in-hospital mortality in patients with COVID-19: a systematic review and meta-analysis. Heart. https://doi.org/10. 1136/heartjnl-2020-317062

3. Majure DT, Gruberg L, Saba SG, Kvasnovsky C, Hirsch JS, Jauhar R (2021) Northwell Health COVID-19 research consortium. Usefulness of elevated troponin to predict death in patients with COVID-19 and myocardial injury. Am J Cardiol 138:100-106

4. Lala A, Johnson KW, Januzzi JL, Russak AJ, Paranjpe I, Richter F, Zhao S, Somani S, Van Vleck T, Vaid A, Chaudhry F, De Freitas JK, Fayad ZA, Pinney SP, Levin M, Charney A, Bagiella E, Narula J, Glicksberg BS, Nadkarni G, Mancini DM, Fuster V (2020) Mount sinai COVID informatics center. Prevalence and impact of myocardial injury in patients hospitalized with COVID19 infection. J Am Coll Cardiol 76:533-546

5. Giustino G, Croft LB, Stefanini GG, Bragato R, Silbiger JJ, Vicenzi M, Danilov T, Kukar N, Shaban N, Kini A, Camaj A, Bienstock SW, Rashed ER, Rahman K, Oates CP, Buckley S, Elbaum LS, Arkonac D, Fiter R, Singh R, Li E, Razuk V, Robinson SE, Miller M, Bier B, Donghi V, Pisaniello M, Mantovani R, Pinto G, Rota I et al (2020) Characterization of myocardial injury in patients with COVID-19. J Am Coll Cardiol 76:2043-2055
6. Bolignano D, Cabassi A, Fiaccadori E, Ghigo E, Pasquali R, Peracino A, Peri A, Plebani M, Santoro A, Settanni F, Zoccali C (2014) Copeptin (CTproAVP), a new tool for understanding the role of vasopressin in pathophysiology. Clin Chem Lab Med 52:1447-1456

7. Struck J, Morgenthaler NG, Bergmann A (2005) Copeptin, a stable peptide derived from the vasopressin precursor, is elevated in serum of sepsis patients. Peptides 26:2500-2504

8. Kolditz M, Halank M, Schulte-Hubbert B, Bergmann S, Albrecht S, Höffken G (2012) Copeptin predicts clinical deterioration and persistent instability in community-acquired pneumonia. Respir Med 106:1320-1328

9. Müller B, Morgenthaler N, Stolz D, Schuetz P, Müller C, Bingisser R, Bergmann A, Tamm M, Christ-Crain M (2007) Circulating levels of copeptin, a novel biomarker, in lower respiratory tract infections. Eur J Clin Invest 37:145-152

10. Koch A, Yagmur E, Hoss A, Buendgens L, Herbers U, Weiskirchen R, Koek GH, Trautwein C, Tacke F (2018) Clinical relevance of copeptin plasma levels as a biomarker of disease severity and mortality in critically ill patients. J Clin Lab Anal 32:e22614

11. Tentzeris I, Jarai R, Farhan S, Perkmann T, Schwarz MA, Jakl G, Wojta J, Huber K (2011) Complementary role of copeptin and high-sensitivity troponin in predicting outcome in patients with stable chronic heart failure. Eur J Heart Fail 13:726-733

12. Khan SQ, Dhillon OS, O'Brien RJ, Struck J, Quinn PA, Morgenthaler NG, Squire IB, Davies JE, Bergmann A, Ng LL (2007) C-terminal provasopressin (copeptin) as a novel and prognostic marker in acute myocardial infarction: Leicester Acute Myocardial Infarction Peptide (LAMP) study. Circulation 115:2103-2110

13. Iba T, Connors JM, Levy JH (2020) The coagulopathy, endotheliopathy, and vasculitis of COVID-19. Inflamm Res 69:1181-1189

14. Kuluöztürk M, İn E, Telo S, Karabulut E, Geçkil AA (2021) Efficacy of copeptin in distinguishing COVID-19 pneumonia from community-acquired pneumonia. J Med Virol. https://doi.org/10. $1002 /$ jmv. 26870

15. Gupta RK, Harrison EM, Ho A, Docherty AB, Knight SR, van Smeden M, Abubakar I, Lipman M, Quartagno M, Pius R, Buchan I, Carson G, Drake TM, Dunning J, Fairfield CJ, Gamble C, Green CA, Halpin S, Hardwick HE, Holden KA, Horby PW, Jackson C, Mclean KA, Merson L, Nguyen-Van-Tam JS, Norman L, Olliaro PL, Pritchard MG, Russell CD, Scott-Brown J et al (2021) Development and validation of the ISARIC 4C Deterioration model for adults hospitalised with COVID-19: a prospective cohort study. Lancet Respir Med. https://doi.org/10.1016/S2213-2600(20) 30559-2

16. Kaufmann CC, Ahmed A, Kassem M, Freynhofer MK, Jäger B, Aicher G, Equiluz-Bruck S, Spiel AO, Funk G-C, Gschwantler M, Fasching P, Wojta J, Huber K (2021) Mid-regional pro-atrial natriuretic peptide independently predicts short-term mortality in COVID-19. Eur J Clin Invest. https://doi.org/10.1111/eci.13531

17. https://www.siemens-healthineers.com/laboratory-diagnostics/ assays-by-diseases-conditions/cardiac-assays/cardiac-tropo nin-assays.

18. Afsar B (2017) Pathophysiology of copeptin in kidney disease and hypertension. Clin Hypertens. https://doi.org/10.1186/ s40885-017-0068-y

19. Popovic M, Ebrahimi F, Urwyler SA, Donath MY, Christ-Crain M (2020) The role of IL-1 in the regulation of copeptin in patients with metabolic syndrome. Endocr Connect 9:715-723

20. Lavillegrand J-R, Garnier M, Spaeth A, Mario N, Hariri G, Pilon A, Berti E, Fieux F, Thietart S, Urbina T, Turpin M, Darrivière L, Fartoukh M, Verdonk F, Dumas G, Tedgui A, Guidet B, Maury E, Chantran Y, Voiriot G, Ait-Oufella H (2021) Elevated plasma IL-6 and CRP levels are associated with adverse clinical outcomes 
and death in critically ill SARS-CoV-2 patients: inflammatory response of SARS-CoV-2 patients. Ann Intens Care 11:9

21. Li X, Liu C, Mao Z, Xiao M, Wang L, Qi S, Zhou F (2020) Predictive values of neutrophil-to-lymphocyte ratio on disease severity and mortality in COVID-19 patients: a systematic review and meta-analysis. Crit Care 24:647

22. Katan M, Morgenthaler N, Widmer I, Puder JJ, König C, Müller B, Christ-Crain M (2008) Copeptin, a stable peptide derived from the vasopressin precursor, correlates with the individual stress level. Neuro Endocrinol Lett 29:341-346

23. Lin Q, Fu F, Chen H, Zhu B (2012) Copeptin in the assessment of acute lung injury and cardiogenic pulmonary edema. Respir Med 106:1268-1277

24. Waldsperger H, Biener M, Stoyanov KM, Vafaie M, Katus HA, Giannitsis E, Mueller-Hennessen M (2020) Prognostic value of elevated copeptin and high-sensitivity cardiac troponin $\mathrm{T}$ in patients with and without acute coronary syndrome: The ConTrACS Study. J Clin Med. https://doi.org/10.3390/jcm9113627

25. Hellenkamp K, Schwung J, Rossmann H, Kaeberich A, Wachter R, Hasenfuß G, Konstantinides S, Lankeit M (2015) Risk stratification of normotensive pulmonary embolism: prognostic impact of copeptin. Eur Respir J Eur Respir Soc 46:1701-1710

26. Potocki M, Reichlin T, Thalmann S, Zellweger C, Twerenbold R, Reiter M, Steuer S, Bassetti S, Drexler B, Stelzig C, Freese M, Winkler K, Haaf P, Balmelli C, Hochholzer W, Osswald S, Mueller C (2012) Diagnostic and prognostic impact of copeptin and high-sensitivity cardiac troponin $\mathrm{T}$ in patients with pre-existing coronary artery disease and suspected acute myocardial infarction. Heart 98:558-565

27. Giannitsis E, Slagman A, Hamm CW, Gehrig S, Vollert JO, Huber K (2020) Copeptin combined with either non-high sensitivity or high sensitivity cardiac troponin for instant rule-out of suspected non-ST segment elevation myocardial infarction. Biomarkers 25:649-658

28. Giannitsis E, Huber K, Hamm CW, Möckel M (2021) Instant ruleout of suspected non-ST-segment elevation myocardial infarction using high-sensitivity cardiac troponin $\mathrm{T}$ with Copeptin versus a single low high-sensitivity cardiac troponin T: findings from a large pooled individual data analysis on 10,329 patients. Clin Res Cardiol 110:194-199

29. Giannitsis E, Clifford P, Slagman A, Ruedelstein R, Liebetrau C, Hamm C, Honnart D, Huber K, Vollert JO, Simonelli C, Schröder M, Wiemer JC, Mueller-Hennessen M, Schroer H, Kastner K, Möckel M (2019) Multicentre cross-sectional observational registry to monitor the safety of early discharge after rule-out of acute myocardial infarction by copeptin and troponin: the Pro-Core registry. BMJ Open 9:e028311

30. Mueller C, Möckel M, Giannitsis E, Huber K, Mair J, Plebani M, Thygesen K, Jaffe AS, Lindahl B (2018) ESC study group on biomarkers in cardiology of the acute cardiovascular care association. Use of copeptin for rapid rule-out of acute myocardial infarction. Eur Heart J Acute Cardiovasc Care 7:570-576

31. Mueller C, Giannitsis E, Möckel M, Huber K, Mair J, Plebani M, Thygesen K, Jaffe AS, Lindahl B (2017) Biomarker Study group of the ESC acute cardiovascular care association. Rapid rule out of acute myocardial infarction: novel biomarker-based strategies. Eur Heart J Acute Cardiovasc Care 6:218-222

32. Möckel M, Searle J, Hamm C, Slagman A, Blankenberg S, Huber K, Katus H, Liebetrau C, Müller C, Muller R, Peitsmeyer P, von Recum J, Tajsic M, Vollert JO, Giannitsis E (2015) Early discharge using single cardiac troponin and copeptin testing in patients with suspected acute coronary syndrome (ACS): a randomized, controlled clinical process study. Eur Heart J 36:369-376

33. Mueller C, Giannitsis E, Jaffe AS, Huber K, Mair J, Cullen L, Hammarsten O, Mills NL, Möckel M, Krychtiuk K, Thygesen K, Lindahl B (2021) ESC Study group on biomarkers in cardiology of the acute cardiovascular care association. Cardiovascular biomarkers in patients with COVID-19. Eur Heart J Acute Cardiovasc Care 10:310-319 